Provided for non-commercial research and education use. Not for reproduction, distribution or commercial use.

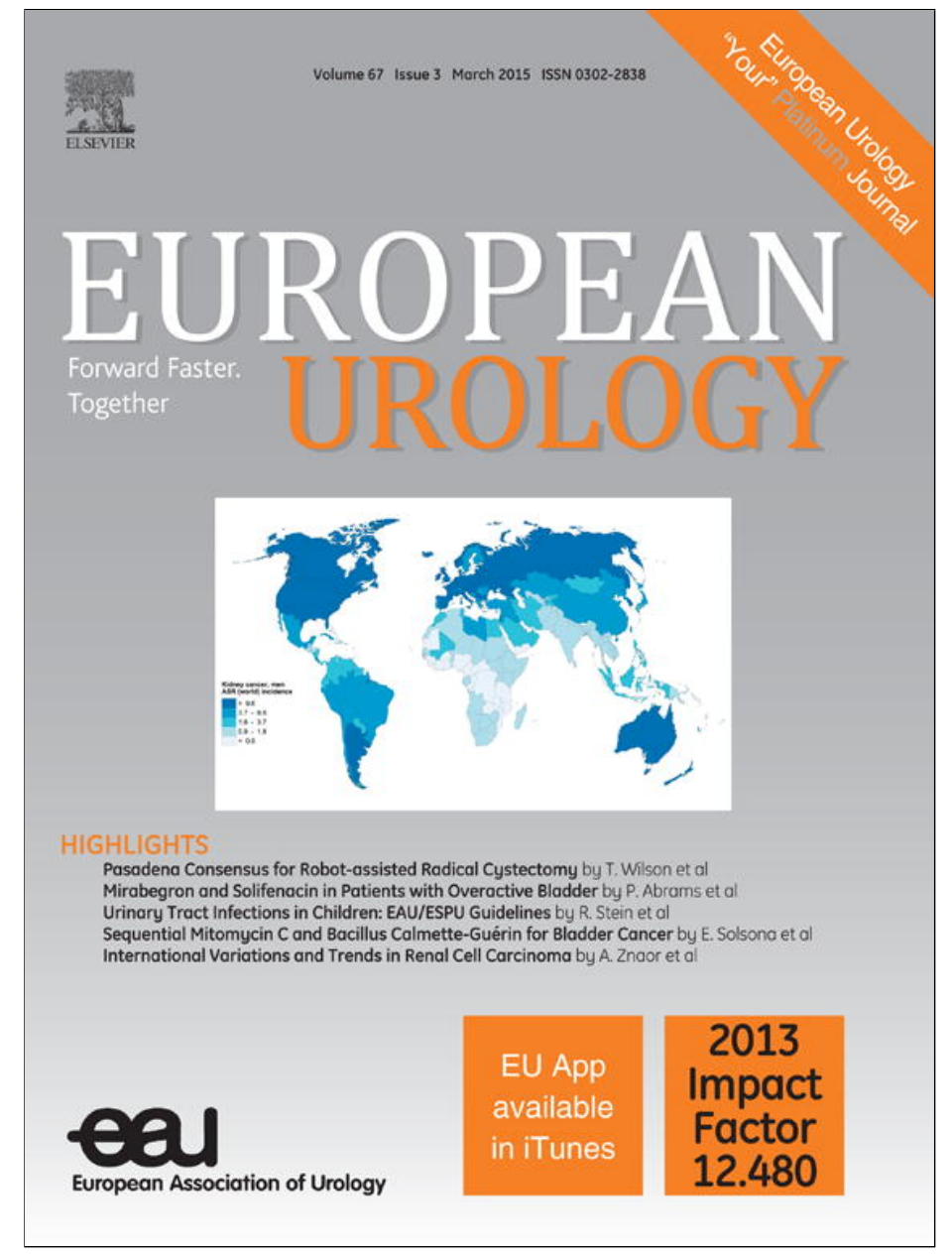

This article appeared in a journal published by Elsevier. The attached copy is furnished to the author for internal non-commercial research and education use, including for instruction at the authors institution and sharing with colleagues.

Other uses, including reproduction and distribution, or selling or licensing copies, or posting to personal, institutional or third party websites are prohibited.

In most cases authors are permitted to post their version of the article (e.g. in Word or Tex form) to their personal website or institutional repository. Authors requiring further information regarding Elsevier's archiving and manuscript policies are encouraged to visit:

http://www.elsevier.com/authorsrights 


\section{Reply to Vincenzo Ficarra, Vito Palumbo, Afrovita Kungulli and Gianluca Giannarini's Letter to the Editor re: Andrea Minervini, Marco Carini, Robert G. Uzzo, Riccardo Campi, Marc C. Smaldone, Alexander Kutikov. Standardized Reporting of Resection Technique During Nephron-sparing Surgery: The Surface-Intermediate- Base Margin Score. Eur Urol 2014;66:803-5}

We read, with great interest, the thoughtful letter by Ficarra et al [1] regarding our recently proposed surface-intermediate-base (SIB) score for objectifying surgical technique reporting during nephron-sparing surgery (NSS) [2]. Some of the authors' concerns address specific technical aspects of the SIB score assignment, whereas others offer opinions regarding the premise and purpose of the novel clinical research tool that we proposed. We thank Ficarra and colleagues for engaging us to clarify some of the nuances of the scoring system.

Prior to our report, there were no standardised definitions for reporting NSS resection techniques in the literature. This void undermines objective and meaningful comparisons of outcomes between surgeons and institutions performing NSS. Indeed, many perioperative and postoperative outcomes are inherently influenced by the kidney surgeon's resection strategy [3]. Consequently, standardised reporting of surgical techniques is essential.

Below we address each of Ficarra and colleagues' concerns.

\section{Response to comment 1}

Ficarra and colleagues raise concerns that the scoring system is assigned visually and not pathologically. Although a histopathologic reporting system, theoretically, may represent the ideal strategy for standardised quantification of resection techniques, such a system is largely prohibitive logistically, as is evident from the current scarcity of granular data on details of resection technique across surgical series. We strongly believe that a surgeon-based, visually assigned approach will substantively lower barriers for consistent reporting and robust data collection. The healthy renal margin beyond the tumour pseudocapsule is the cornerstone of the SIB scoring system. Visual anatomic grading of surgical margins by the surgeon immediately following resection allows unity of cues from appropriate specimen orientation, tumour contour, pseudocapsule integrity, and correspondence of the specimen to anatomic landmarks on the tumour bed. Arguably, no one is better able to differentiate between enucleation, enucleoresection, and resection in a given specimen than the surgeon (Fig. 1A and 1B) [4]. Ficarra et al raised concerns that the thickness of healthy renal parenchyma and the integrity of the pseudocapsule are extremely difficult to ascertain visually. We believe these concerns are not justified. Enucleation, enucleoresection, resection, and capsulotomy, as part of the SIB score assignment, are defined based on assessment of the tumour's contours rather than on speculative evaluation of the margin thickness. We submit that all experienced renal surgeons are able to visually recognise the peritumoural pseudocapsule and its possible violation (capsulotomy) with high fidelity. Moreover, the proposed quantification of partial nephrectomy (PN) resection technique is extremely specific, harnessing the closest margin to the pseudocapsule in each designated region of resection (Fig. 1C). In this context, the concern about "leopard spots" of thin healthy renal tissue beyond the pseudocapsule, described by Minervini et al [5] and discussed by Ficarra and colleagues, would not alter the final SIB score.

\section{Response to comments 2-4}

Ficarra and colleagues raise concerns regarding how the surface, intermediate, and base tumour surfaces are defined. Unfortunately, the figure presented by Ficarra et al largely misinterprets our initial proposal. To clarify, a complete overview of the SIB surface assignment is shown in Figure 2. The surface, intermediate, and base areas are not influenced by the anatomic location of the tumour or by the depth of its penetration into normal parenchyma. Regardless of both polar location and depth of intrarenal growth, the SIB surfaces are defined as the circumferential surfaces of the intrarenal component, dividing each into approximately three equal slices. By definition, it is always possible to divide the tumour bed into these three areas. Moreover, the circumferential analysis allows for optimal overall visualisation of the resection technique without omitting 

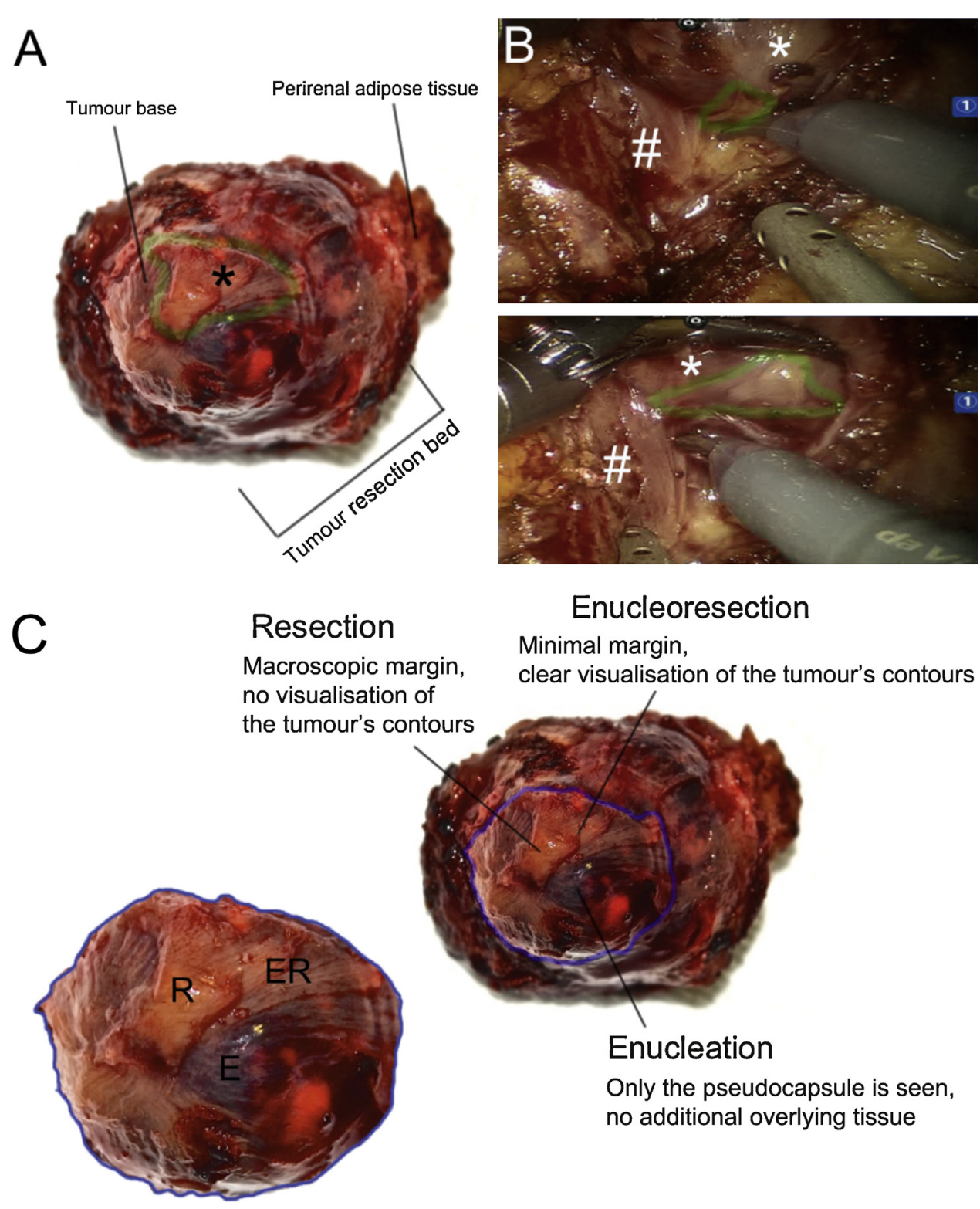

\section{Enucleoresection}

Minimal margin,

clear visualisation of the tumour's contours

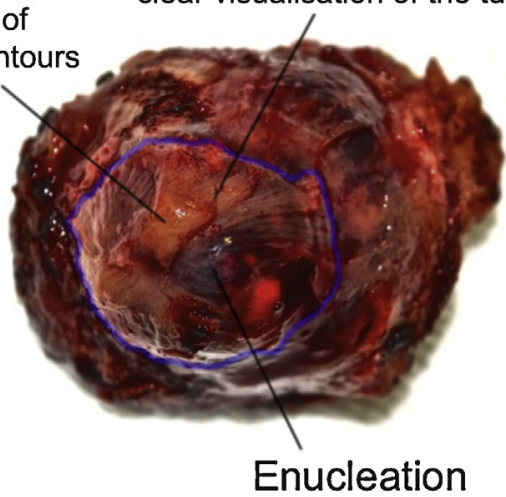

Only the pseudocapsule is seen, no additional overlying tissue

Fig. 1 - Definitions of partial nephrectomy (PN) resection techniques. (A) The intrarenal portion of the tumour is shown facing the viewer. The perirenal adipose tissue is visible on the far side of the tumour (slightly out of focus). (B) Intraoperative screenshots taken during a robot-assisted PN procedure demonstrate two time points of resection. The correlation between intraoperative findings and anatomic characteristics of the resection margin can be readily appreciated. ${ }^{*}$ Tumour. \# Tumour bed. (C) Visual analysis of the resection margin at tumour base: Three zones within the deepest area of the resection bed (base) are highlighted. Enucleation, enucleoresection, and resection are defined based on the ability to macroscopically appreciate the tumour's contours and the visibility of the peritumoural pseudocapsule. Although resection and enucleoresection zones are visible, presence of a zone of enucleation results in the designation of a score of 0 (enucleation) for this area (base).

$E$ = enucleation; $E R=$ enucleoresection; $R$ = resection.

any tumour surfaces. Importantly, the location at which the surgeon begins the resection should not influence the SIB score (Fig. 3). Ficarra and colleagues' concern regarding cutting the tumour in the operating room and thus compromising margin status is unfounded because the score is visually assigned by the surgeon based on examination of the tumour surface.

\section{Response to comments 5-7}

Ficarra and colleagues raised a concern that a score of 0 on the surface component is not possible because, during pure enucleation, "an initial incision in the renal capsule is performed a few millimetres away from the tumour before blunt development of the natural plane between the pseudocapsule and healthy parenchyma" is developed [6]. Again, we stress that the SIB score pivots on the minimal margin present within each designated SIB area. As such, as long as normal parenchyma does not circumferentially cover more than one-third of the tumour's resected surface, a score of 0 will be assigned to the surface component of the SIB score during an attempted pure enucleation (Figs. 1-3). Furthermore, Ficarra and colleagues raised a concern that some SIB score combinations may be 

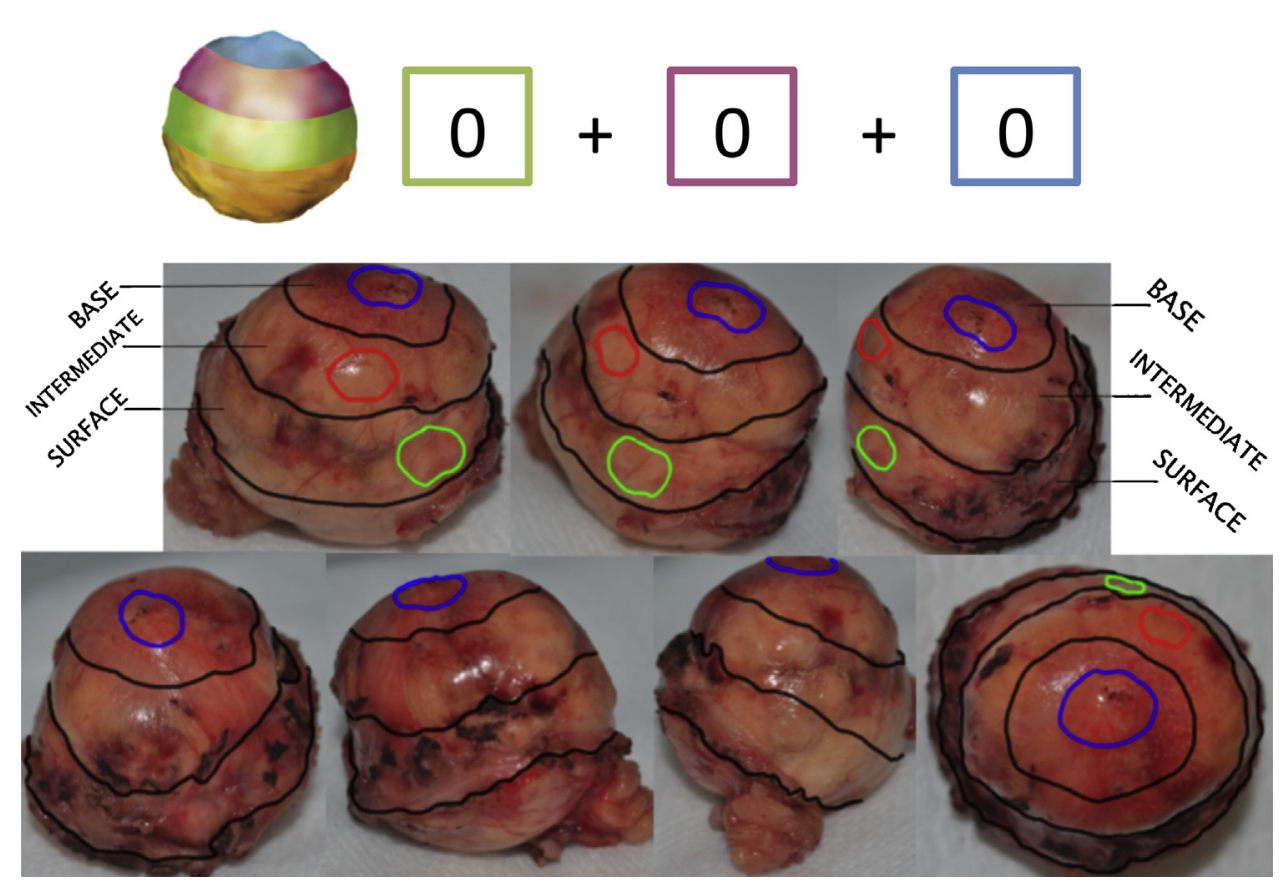

Fig. 2 - Overview of the main steps for surface-intermediate-base (SIB) score assignment: $0+0+0=0$ (pure enucleation). The tumour resection bed is ideally seen as a hemisphere, and its surface is divided into three roughly equal circumferential areas of approximately the same height (each onethird of the global height): the surface, intermediate, and base areas. Regardless of the surgeon's resection strategy, these areas are circumferentially analysed to identify the macroscopically evident zone of minimal margin. This will be the specific zone for the score assignment in each area. This zone must be visually detectable but not microscopic. No percentages must be reported. In the image, the specimen is turned in a clockwise direction to show the 360 visual analysis. In the case presented, the margin thickness is homogeneous within all three areas; only the pseudocapsule is seen without any additional overlying tissue; thus, the score is $0+0+0$. The SIB score sum $(0+0+0=0)$ and, consequently, the definition of resection technique (pure enucleation) are obtained (Table 1 of Minervini et al [2]).

more prevalent clinically than others and that the SIB score reporting is too granular. Indeed, the SIB score proposal lists all possible combination of scores and does not attempt to stratify them by probability of occurrence in clinical practice. We agree with Ficarra and colleagues that realworld clinical data are necessary to determine which combinations are most prevalent, and we are working to develop a clinical data set that gleans insight into this issue. The thickness of the healthy renal margin is often nonhomogeneous over the tumour resection bed after PN. Such hybrid resection techniques are not captured by current reporting methods. In fact, these shortcomings of the current state of NSS-technique reporting catalysed the international collaboration to propose the SIB scoring system. Whether or not differences in the SIB score are clinically relevant can be determined only from future analyses of clinical data.

We acknowledge that the SIB score focuses only on parameters relating to tumour resection. That is the sole purpose of the SIB score. Some of our future work will focus on assessing associations between short- and long-term outcomes and the SIB score, relationships between tumour complexity and strategy of resection, and the effect of various resection techniques on postoperative renal function.

Finally, we have extensively tested various hypotheses in the clinical setting with regard to how best to define consistent anatomic landmarks for each resection technique and the most appropriate classification model. The SIB scoring system represents the final outcome of a systematic study of PN rather than a trial-and-error attempt.

Ficarra et al reference the European Association of Urology guidelines [7] and state their opinion that it "is sufficient and simpler to distinguish minimal PN from traditional, more extensive wedge resection." Certainly, we value the opinion of these experts; however, the guidelines are a dynamic document based on the latest clinical data. We submit that thoughtful analyses of robust granular data can afford not only insights into current practices but also opportunities for potentially improving the care of our patients. Indeed, clinical research attempts to overcome the limitations of available knowledge. Only $5 \mathrm{yr}$ ago there was no standardised system to describe the anatomic characteristics of renal tumours; today, the nephrometric scores represent the cornerstone of the preoperative assessment of renal neoplasms and afford standardised communication between renal surgeons. We hope that the merits of future work will determine whether the SIB score represents a meaningful contribution to the urologic literature. As such, a prospective, single-centre study validating the surgeonbased SIB score assignments is nearing completion, and its preliminary results showed good statistical correlations with histopathologic findings.

A prospective, multicentre study to assess the utility of the SIB score and the surgeon assessment of preserved volume metric [8] was initiated recently at 12 European and 3 US centres. We hope that the evaluation of the SIB score in a clinical setting by an international group of experts will 

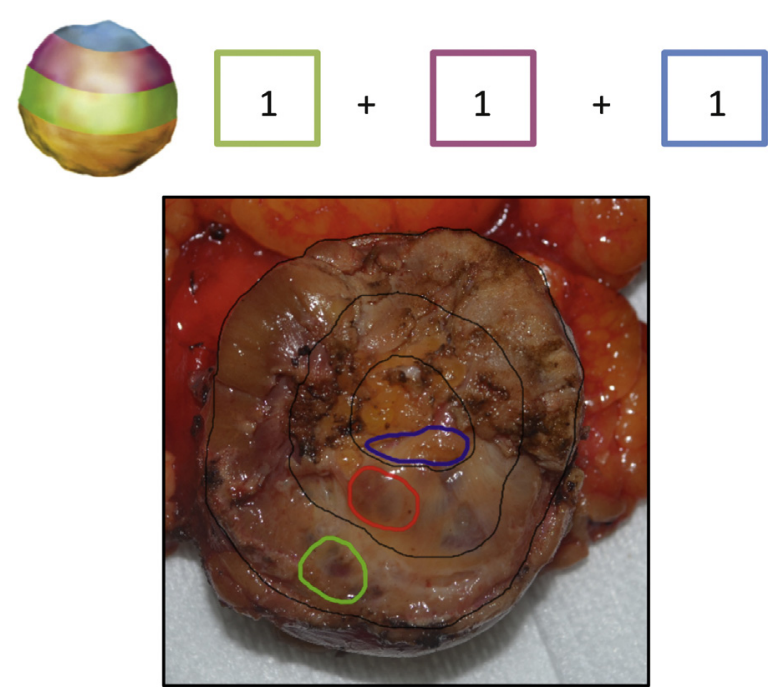

Fig. 3 - Overview of the main steps for surface-intermediate-base (SIB) score assignment: $1+1+1=3$ (pure enucleoresection). In this figure, the margin thickness is not homogeneous within the three areas. The healthy renal margin is thicker on one side of the tumour resection bed (top of image) and thinner on the other (bottom of image). Each area is carefully analysed circumferentially, and the zone of minimal margin is identified. In this case, one point is assigned to the zones of minimal margin within the surface, intermediate, and base areas because they meet the definition of enucleoresection (presence of a minimal margin of healthy parenchyma that allows for clear visualisation of the tumour's contours). The SIB score sum is 3; consequently, the definition of the overall resection technique is pure enucleoresection.

address many of the concerns of Ficarra and colleagues. We thank them for their thoughtful feedback.

Conflicts of interest: The authors have nothing to disclose.

\section{References}

[1] Ficarra V, Palumbo V, Kungulli A, Giannarini G. Re: Andrea Minervini, Marco Carini, Robert G. Uzzo, Riccardo Campi, Marc C. Smaldone, Alexander Kutikov. Standardized reporting of resection technique during nephron-sparing surgery: the surface-intermediate-base margin score. Eur Urol 2014;66:803-5, Eur Urol 2015;67: e45-7
[2] Minervini A, Carini M, Uzzo RG, Campi R, Smaldone MC, Kutikov A Standardized reporting of resection technique during nephronsparing surgery: the surface-intermediate-base margin score. Eur Urol 2014;66:803-5.

[3] Mukkamala A, Allam CL, Ellison JS, et al. Tumor enucleation vs sharp excision in minimally invasive partial nephrectomy: technical benefit without impact on functional or oncologic outcomes. Urology 2014;83:1294-9.

[4] Hagemann IS, Lewis Jr JS. A retrospective comparison of 2 methods of intraoperative margin evaluation during partial nephrectomy. J Urol 2009;181:500-5.

[5] Minervini A, Di Cristofano C, Lapini A, et al. Histopathologic analysis of peritumoral pseudocapsule and surgical margin status after tumor enucleation for renal cell carcinoma. Eur Urol 2009;55: 1410-8.

[6] Carini M, Minervini A, Masieri L, et al. Simple enucleation for the treatment of pT1a renal cell carcinoma: our 20-year experience. Eur Urol 2006;50:1263-71.

[7] Ljungberg B, Cowan NC, Hanbury DC, et al. EAU guidelines on renal cell carcinoma: the 2010 update. Eur Urol 2010;58:398-406.

[8] Tobert CM, Boelkins B, Culver S, et al. Surgeon assessment of renal preservation with partial nephrectomy provides information comparable to measurement of volume preservation with 3-dimensional image analysis. J Urol 2014;191:1218-24.

\author{
Andrea Minervini ${ }^{\mathrm{a}, *}$ \\ Riccardo Campi ${ }^{\mathrm{a}}$ \\ Marc C. Smaldone ${ }^{\mathrm{b}}$ \\ Robert G. Uzzo ${ }^{\text {b }}$ \\ Marco Carini ${ }^{\mathrm{a}}$ \\ Alexander Kutikov ${ }^{\mathrm{b}}$
}

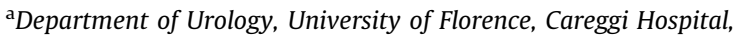
Florence, Italy ${ }^{\mathrm{b}}$ Division of Urologic Oncology, Fox Chase Cancer Center, Philadelphia, PA, USA

${ }^{*}$ Corresponding author. Clinica Urologica I, Azienda Ospedaliera Careggi, Università di Firenze, Viale San Luca, 50134 Firenze, Italy. Tel. +390552758011, +390557949209, +393475865716; Fax: +390552758014 E-mail address: andreamine@libero.it (A. Minervini).

October 30, 2014 